TRANSACTIONS OF THE

AMERICAN MATHEMATICAL SOCIETY

Volume 350, Number 7, July 1998, Pages 2953-2972

$\mathrm{S} 0002-9947(98) 02045-5$

\title{
SUM THEOREMS FOR MONOTONE OPERATORS AND CONVEX FUNCTIONS
}

\author{
S. SIMONS
}

\begin{abstract}
In this paper, we derive sufficient conditions for the sum of two or more maximal monotone operators on a reflexive Banach space to be maximal monotone, and we achieve this without any renorming theorems or fixed-pointrelated concepts. In the course of this, we will develop a generalization of the uniform boundedness theorem for (possibly nonreflexive) Banach spaces. We will apply this to obtain the Fenchel Duality Theorem for the sum of two or more proper, convex lower semicontinuous functions under the appropriate constraint qualifications, and also to obtain additional results on the relation between the effective domains of such functions and the domains of their subdifferentials. The other main tool that we use is a standard minimax theorem.
\end{abstract}

\section{INTRODUCTION}

The primary purpose of this paper is to find sufficient conditions for the sum of two or more maximal monotone operators on a reflexive Banach space to be maximal monotone, and to achieve this without any renorming theorems or fixedpoint-related concepts. In the course of this, we will develop a generalization of the uniform boundedness theorem for general (i.e., possibly nonreflexive) Banach spaces, which we will also apply to obtain the Fenchel Duality Theorem for the sum of two or more proper, convex lower semicontinuous functions under the appropriate constraint qualifications.

Let $E$ be a (nontrivial) Banach space. If $S: E \rightarrow 2^{E^{*}}$, we write

$$
G(S):=\left\{\left(x, x^{*}\right): x \in E, x^{*} \in S x\right\}
$$

and

$$
D(S):=\{x: x \in E, S x \neq \emptyset\} .
$$

$S$ is said to be monotone if

$$
\left(x, x^{*}\right),\left(y, y^{*}\right) \in G(S) \quad \Longrightarrow \quad\left\langle x-y, x^{*}-y^{*}\right\rangle \geq 0 .
$$

$S$ is said to be maximal monotone if $S$ is monotone, and $S$ has no proper monotone extension. This is equivalent to the statement

$$
\left(z, z^{*}\right) \in E \times E^{*} \text { and } \inf _{\left(s, s^{*}\right) \in G(S)}\left\langle s-z, s^{*}-z^{*}\right\rangle \geq 0 \quad \Longrightarrow \quad\left(z, z^{*}\right) \in G(S) .
$$

Received by the editors July 16, 1996.

1991 Mathematics Subject Classification. Primary 47H05, 46B10; Secondary 49J35, 46A30.

Key words and phrases. Banach space, reflexivity, maximal monotone operator, sum theorem, constraint qualification, proper convex lower semicontinuous function, uniform boundedness theorem, Fenchel Duality Theorem, minimax theorem. 
If $S_{1}, S_{2}: E \rightarrow 2^{E^{*}}$, we define $S_{1}+S_{2}: E \rightarrow 2^{E^{*}}\left(\right.$ with $\left.D\left(S_{1}+S_{2}\right)=D\left(S_{1}\right) \cap D\left(S_{2}\right)\right)$ by

$$
\left(S_{1}+S_{2}\right) x:=S_{1} x+S_{2} x .
$$

It is obvious that if $S_{1}$ and $S_{2}$ are monotone then $S_{1}+S_{2}$ is also monotone. It is not true that if $S_{1}$ and $S_{2}$ are maximal monotone then $S_{1}+S_{2}$ is necessarily maximal monotone. In order to guarantee this, one has to assume that the operators satisfy a "constraint qualification". We refer the reader to the discussion on p. 54 of [14]. The "sum theorem" of Rockafellar (see [18], Theorem 1, p. 76) asserts that if $E$ is reflexive, $S_{1}, S_{2}: E \rightarrow 2^{E^{*}}$ are maximal monotone and

$$
D\left(S_{1}\right) \cap \operatorname{int} D\left(S_{2}\right) \neq \emptyset,
$$

then $S_{1}+S_{2}$ is maximal monotone. This result has recently been generalized by Attouch-Riahi-Théra (see [2], Théorème 4), who proved that (0.1) can be weakened to

$$
D\left(S_{1}\right)-D\left(S_{2}\right) \quad \text { is absorbing. }
$$

(A subset $A$ of $E$ is said to be absorbing if $\bigcup_{\lambda>0} \lambda A=E$.) It will follow from the results in this paper that $(0.2)$ can be further weakened to

$$
\operatorname{co} D\left(S_{1}\right)-\operatorname{co} D\left(S_{2}\right) \text { is absorbing, }
$$

where "co" stands for "convex hull of". In fact, we shall show how to associate a proper, convex lower semicontinuous function $\chi_{S}$ to each monotone operator $S: E \rightarrow 2^{E^{*}}$ for which $D(S) \neq \emptyset$ in such a way that $\operatorname{co} D(S) \subset \operatorname{dom} \chi_{S}$, and we shall prove in Corollary 20 that (0.3) can be further weakened to the "dom-dom" constraint qualification:

$$
\operatorname{dom} \chi_{S_{1}}-\operatorname{dom} \chi_{S_{2}} \text { is absorbing. }
$$

Parenthetically, there is no prima facie reason that (0.4) should imply that $D\left(S_{1}\right) \cap$ $D\left(S_{2}\right) \neq \emptyset$, though it will follow from Theorem 19 that this implication is true if $E$ is reflexive and $S_{1}$ and $S_{2}$ are maximal monotone.

In fact, there are also "subspace" versions of the above constraint qualifications that are known to be sufficient. Attouch-Riahi-Théra have proved (see [2], Corollaire 1) that (0.2) can be weakened to

$$
\bigcup_{\lambda>0} \lambda\left[D\left(S_{1}\right)-D\left(S_{2}\right)\right]=\overline{\operatorname{lin}\left(D\left(S_{1}\right)-D\left(S_{2}\right)\right)},
$$

where "lin" stands for "linear span of", while Chu has proved (see [10], Corollary $3.5)$ that (0.1) can be weakened to

$$
\left\{\begin{array}{r}
\operatorname{co} D\left(S_{1}\right)-\operatorname{co} D\left(S_{2}\right) \text { is a neighborhood of } 0 \\
\text { relative to } \frac{\operatorname{lin}\left(D\left(S_{1}\right)-D\left(S_{2}\right)\right)}{} .
\end{array}\right.
$$

In Definition 22, we shall introduce a new concept, the " $\chi$ constraint qualification", and we shall prove in Theorem 26 that if $E$ is reflexive and $S_{1}, S_{2}: E \rightarrow 2^{E^{*}}$ are maximal monotone operators satisfying the $\chi$ constraint qualification then $S_{1}+S_{2}$ is maximal monotone. We shall show in Lemma 23 that if

$$
\bigcup_{\lambda>0} \lambda\left[\operatorname{co} D\left(S_{1}\right)-\operatorname{co} D\left(S_{2}\right)\right]=\overline{\operatorname{lin}\left(D\left(S_{1}\right)-D\left(S_{2}\right)\right)},
$$


then $S_{1}, S_{2}$ satisfy the $\chi$ constraint qualification, so Theorem 26 generalizes both [2], Corollaire 1 and [10], Corollary 3.5. ${ }^{1}$

We now give a brief discussion of the main results of the paper. Corollary 4 contains a "second order" generalization of the uniform boundedness theorem see Remark 6 for more on this. In fact, if we are only interested in finding a simple proof of Rockafellar's original result (that is, using the condition (0.1)), it suffices to have Corollary 5, which follows from the standard result that a convex lower semicontinuous function is locally bounded on the interior of its domain.

We give the formal definition and some elementary properties of $\chi_{S}$ in Definition 8 and Lemmas 9-11. The function $\chi_{S}$ was originally introduced in [9]. In the interest of keeping this paper as self-contained as possible, we reprove a couple of the simpler results from [9] - collecting in Remark 12 statements of those results in [9] that originally motivated the introduction of $\chi_{S}$.

Lemmas 13 and 15 are our main results about monotone operators that satisfy the dom-dom constraint qualification (0.4). Lemma 13 is a rather technical result, in which we establish the existence of a bound $M$ in terms of an auxiliary function $f$. At first sight, the function $f$ seems to be "pulled out of a hat". In fact this is not the case - we shall explain in Remark 21 that the definition of $f$ is in fact forced by the problem that we are considering. We assume for the first time that $E$ is reflexive in the penultimate line of Lemma 13. Lemma 13 leads rapidly to Lemma 15 , in which we use a minimax theorem (see below for more on this).

In Lemmas 16 and 17, we impose the additional condition that $S_{1}$ and $S_{2}$ be maximal monotone. It is in Lemma 17 that we establish for the first time the existence of an element of $G\left(S_{1}+S_{2}\right)$ satisfying certain properties. We bootstrap this result, first by a translation in $E$ in Lemma 18 , and subsequently by a translation in $E^{*}$ in Theorem 19. It is then but a short step to the proof in Corollary 20 that if the dom-dom constraint qualification (0.4) is satisfied, then $S_{1}+S_{2}$ is maximal monotone.

We introduce the $\chi$ constraint qualification formally in Definition 22. We perform a further bootstrapping operation by restriction to a subspace in order to establish the result in Theorem 26 that if $S_{1}, S_{2}$ satisfy the $\chi$ constraint qualification, then $S_{1}+S_{2}$ is maximal monotone. This bootstrapping result, which is not entirely trivial, appears in Lemma 25. We point out for the record that Lemma 25 is valid if $E$ is any normed space.

Conditions of "dom-dom" type were first introduced by Borwein and AttouchBrézis in a different context - that of finding weakened constraint conditions for the validity of the Fenchel Duality Theorem on the conjugate of the sum of proper, convex lower semicontinuous functions on a Banach space. In the final section of this paper we show how Theorem 3 and the minimax technique lead to simple "geometric" proofs of the main results from [1]. Both the theory of maximal monotone operators and the theory of the Fenchel Duality Theorem concern duality in a fundamental way. It is, therefore, worth noting that the unifying result, Theorem 3, does not concern duality - the dual space of $F$ is not mentioned. We close the paper by showing how Corollary 31 can be combined with Corollary 4 to extend to the nonreflexive case a recent result of Borwein.

\footnotetext{
${ }^{1}$ We refer the reader to the "Remark added in press" at the end of this paper for more information about the constraint qualifications discussed above.
} 
We conclude this introduction with a few words about what we use and what we do not use in this paper. In Lemma 15, we use the minimax theorem, Theorem 14, which follows from a result of Fan. It is important to note that the set $A$ in Theorem 14 is not topologized. This use of a minimax theorem is actually for notational convenience. It would be also possible to obtain Lemma 15 from Lemma 13 and the Eidelheit Separation Theorem or a Sandwich Theorem, but the technical details would be much more complicated. We can make a similar comment about the use of Theorem 14 in Lemma 33. The technique used to prove Lemma 15 and Lemma 17 is a more involved version of that used in [20], Remark 7, to prove that if $E$ is reflexive and $S: E \rightarrow 2^{E^{*}}$ is maximal monotone, then $S+J$ is surjective (where $J$ is the duality map), coupled with a technique due to Minty and Browder (see [8], Lemma 6, p. 99). Under the appropriate conditions the converse of the above surjectivity result is true, i.e., if $S: E \rightarrow 2^{E^{*}}$ is monotone and $S+J$ is surjective then $S$ is maximal monotone. Indeed, the analysis in [18], [2] and [10] relies heavily on this fact. However, in order for this to be true, it seems to be necessary to renorm $E$. The renorming theorem is highly nontrivial and, as we have already pointed out, the analysis in this paper does not depend on any renorming theorems - see Remark 21 for an explanation of how this is achieved. We also reiterate that the analysis in this paper does not depend on any fixed-point theorems either.

The bounds required in our analysis depend ultimately on the Baire Category Theorem, which is used in Lemma 1. It is also possible to use the minimax technique to generalize other results on the sum of two maximal monotone operators on a reflexive space without the use of renorming or fixed-point theorems, such as the result of Brézis-Crandall-Pazy (see [7]). Here the required bounds are established using techniques that do not depend on the Baire Category Theorem. We do not discuss these results further in this paper, since they would take us too far afield.

\section{A GeneralizATION OF THE UNIFORM BOUNDEDNESS THEOREM}

Lemma 1, which is obviously related to the open mapping theorem, can in fact be generalized considerably. (In this connection, we refer the reader to Robinson [16], Ursescu [21] and Borwein [4]). Here we confine our attention to what we will need in this paper. The idea for Lemma 1 is taken from Aubin-Ekeland [3], Lemma 3.3.9, p. 136 .

Lemma 1. Let $X$ and $Y$ be closed convex subsets of a Banach space $F, X+Y$ be absorbing, and $X$ be bounded. Then $X+Y$ is a neighborhood of 0 in $F$.

Proof. Since $\overline{X+Y}$ is closed, convex and absorbing, it follows from the usual Baire Category Theorem argument that $\overline{X+Y}$ is a neighborhood of 0 in $F$. Choose $\eta>0$ so that

$$
w \in F \text { and }\|w\| \leq 2 \eta \quad \Longrightarrow \quad w \in \overline{X+Y} .
$$

We shall prove that

$$
w \in F \text { and }\|w\| \leq \eta \quad \Longrightarrow \quad w \in X+Y,
$$

which will give the desired result. So let $w \in F$ and $\|w\| \leq \eta$. Then, from (1.1), $2 w \in \overline{X+Y}$; consequently

$$
\text { there exists } z_{1} \in X+Y \text { such that }\left\|2 w-z_{1}\right\| \leq \eta \text {. }
$$


From (1.1) again, $4 w-2 z_{1}=2\left(2 w-z_{1}\right) \in \overline{X+Y}$, and thus

$$
\text { there exists } z_{2} \in X+Y \text { such that }\left\|4 w-2 z_{1}-z_{2}\right\| \leq \eta \text {. }
$$

Continuing this argument, we find $z_{1}, z_{2}, z_{3}, \cdots \in X+Y$ such that, for all $k \geq 1$,

$$
\left\|2^{k} w-2^{k-1} z_{1}-\cdots-z_{k}\right\| \leq \eta
$$

from which

$$
\left\|w-2^{-1} z_{1}-\cdots-2^{-k} z_{k}\right\| \leq 2^{-k} \eta
$$

hence $\sum_{k=1}^{\infty} 2^{-k} z_{k}=w$. For all $n \geq 1$, choose $x_{n} \in X$ and $y_{n} \in Y$ such that $x_{n}+y_{n}=z_{n}$. Since $X$ is bounded, closed and convex, there exists $x \in X$ such that $\sum_{k=1}^{\infty} 2^{-k} x_{k}=x$. Then

$$
\sum_{k=1}^{\infty} 2^{-k} y_{k}=\sum_{k=1}^{\infty} 2^{-k}\left(z_{k}-x_{k}\right)=\sum_{k=1}^{\infty} 2^{-k} z_{k}-\sum_{k=1}^{\infty} 2^{-k} x_{k}=w-x .
$$

Since $Y$ is closed and convex, $w-x \in Y$. This completes the proof of (1.2).

Our first result on convex functions is purely algebraic, and does not use the norm structure of $F$. If $f: F \rightarrow \mathbb{R} \cup\{\infty\}$ is convex and $n \geq 1$, we write

$$
\operatorname{dom} f:=\{x: x \in F, f(x) \in \mathbb{R}\} \quad \text { and } \quad F\{f \leq n\}:=\{x: x \in F, f(x) \leq n\} .
$$

Lemma 2. Let $F$ be a normed space, $f: F \rightarrow \mathbb{R} \cup\{\infty\}$ be a convex function, $Y$ be a subspace of $F$ and $\operatorname{dom} f+Y$ be absorbing. Then there exists $n \geq 1$ such that

$$
F\{f \leq n\}+Y \quad \text { is absorbing. }
$$

Proof. Since $0 \in \operatorname{dom} f+Y$, there exists $z \in \operatorname{dom} f \cap Y$. Let $n \geq(f(z)+1) \vee 0$. We shall show that $n$ has the required property. Let $w$ be an arbitrary element of $F$. Then there exist $\lambda>0$ and $x \in \operatorname{dom} f$ such that $\lambda w \in x+Y$. We choose $\mu \in(0,1]$ so that $\mu(f(x)-n+1) \leq 1$. We shall prove that

$$
\mu \lambda w \in F\{f \leq n\}+Y .
$$

Since we can carry out this construction for all $w \in F$, this will establish (2.1), which will complete the proof of Lemma 2. Indeed,

$$
\mu \lambda w \in \mu[x+Y]=\mu x+Y=\mu x+[(1-\mu) z+Y]=[\mu x+(1-\mu) z]+Y
$$

and, from the choices of $n$ and $\mu$,

$$
f(\mu x+(1-\mu) z) \leq \mu f(x)+(1-\mu)(n-1) \leq n .
$$

This completes the proof of (2.2).

Theorem 3. Let $F$ be a Banach space, $g: F \rightarrow \mathbb{R} \cup\{\infty\}$ be a convex lower semicontinuous function, $Y$ be a closed subspace of $F$ and $\operatorname{dom} g+Y$ be absorbing. Then there exists $n \geq 1$ such that $F\{g \leq n,\|\| \leq n\}+Y$ is a neighborhood of 0 in $F$.

Proof. Write $f:=g \vee\|\|$. Then $\operatorname{dom} f=\operatorname{dom} g$. From Lemma 2 , there exists $n \geq 1$ such that $F\{f \leq n\}+Y$ is absorbing. The required result now follows from Lemma 1 since the set $F\{f \leq n\}=F\{g \leq n,\|\| \leq n\}$ is bounded, closed and convex. 
Corollary 4 is easily deduced from Theorem 3 by writing

$$
F:=E^{2}, \quad Y:=\{(z, z): z \in E\},
$$

with the norm on $F$ given by (for instance) $\left\|\left(x_{1}, x_{2}\right)\right\|:=\left\|x_{1}\right\| \vee\left\|x_{2}\right\|$, and defining $g: F \rightarrow \mathbb{R} \cup\{\infty\}$ by

$$
g\left(x_{1}, x_{2}\right):=g_{1}\left(x_{1}\right) \vee\left\|x_{1}\right\| \vee g_{2}\left(x_{2}\right) \vee\left\|x_{2}\right\| .
$$

Corollary 4. Let $E$ be a Banach space, $g_{1}, g_{2}: E \rightarrow \mathbb{R} \cup\{\infty\}$ be convex, lower semicontinuous functions and $\operatorname{dom} g_{1}-\operatorname{dom} g_{2}$ be absorbing. Then there exists $n \geq 1$ such that

$$
E\left\{g_{1} \leq n,\|\| \leq n\right\}-E\left\{g_{2} \leq n,\|\| \leq n\right\} \quad \text { is a neighborhood of } 0 .
$$

In particular:

Corollary 5. Let $E$ be a Banach space, $g_{1}, g_{2}: E \rightarrow \mathbb{R} \cup\{\infty\}$ be convex, lower semicontinuous functions and $\operatorname{dom} g_{1} \cap \operatorname{int}\left(\operatorname{dom} g_{2}\right) \neq \emptyset$. Then there exists $n \geq 1$ such that (4.1) is satisfied.

Proof. Of course, this result follows from Corollary 4. However, it also follows from the more standard result that a convex lower semicontinuous function is locally bounded on the interior of its domain. (See Phelps [14], Proposition 3.3, p. 39.) Let $u \in \operatorname{dom} g_{1} \cap \operatorname{int}\left(\operatorname{dom} g_{2}\right)$. Then there exist $\eta>0$ and $m \geq 1$ such that

$$
w \in E \text { and }\|w\| \leq \eta \quad \Longrightarrow \quad\left(g_{2} \vee\|\|\right)(u-w) \leq m .
$$

We now take $n \geq g_{1}(u) \vee\|u\| \vee m$. If $w \in E$ and $\|w\| \leq \eta$, then the identity $w=u-(u-w)$ shows that $w \in E\left\{g_{1} \leq n,\|\| \leq n\right\}-E\left\{g_{2} \leq n,\|\| \leq n\right\}$, which gives the required result.

Remark 6. Suppose we take $g_{2}$ to be the indicator function of $\{0\}$, i.e.,

$$
g_{2}(x):=\left\{\begin{aligned}
0 & (x=0) \\
\infty & (x \neq 0)
\end{aligned}\right.
$$

Then Corollary 4 implies the following result: Let $g_{1}: E \rightarrow \mathbb{R}$ be a convex, lower semicontinuous function and $\operatorname{dom} g_{1}$ be absorbing. Then there exist $\eta>0$ and $n \geq 1$ such that

$$
w \in E \text { and }\|w\| \leq \eta \quad \Longrightarrow \quad g_{1}(w) \leq n .
$$

Now let $F$ be a normed space and $\mathcal{B}$ be a nonempty pointwise bounded set of continuous linear maps from $E$ into $F$. Define $g_{1}: E \rightarrow \mathbb{R}$ by

$$
g_{1}(x):=\sup _{T \in \mathcal{B}}\|T x\| .
$$

It then follows from (6.1) that

$$
T \in \mathcal{B} \quad \Longrightarrow \quad\|T\| \leq \frac{n}{\eta} .
$$

It is this observation that justifies our description of Corollary 4 as a "second order" generalization of the uniform boundedness theorem. The proof of the uniform boundedness theorem given above can be found in Holmes [12], §17, p. 134. 
The FUnCTION $\chi_{S}$

We suppose throughout this section that $E$ is a Banach space. We write

$$
\begin{aligned}
& \mathcal{C}\left(E \times E^{*}\right) \\
& \quad:=\left\{a: a \in[0, \infty)^{E \times E^{*}}, a \text { is finitely nonzero and } \sum_{\left(s, s^{*}\right) \in E \times E^{*}} a_{\left(s, s^{*}\right)}=1\right\},
\end{aligned}
$$

and define affine maps $p: \mathcal{C}\left(E \times E^{*}\right) \rightarrow E, q: \mathcal{C}\left(E \times E^{*}\right) \rightarrow E^{*}$ and $r: \mathcal{C}\left(E \times E^{*}\right) \rightarrow \mathbb{R}$ by

$$
\begin{aligned}
p(a) & :=\sum_{\left(s, s^{*}\right) \in \mathcal{C}\left(E \times E^{*}\right)} a_{\left(s, s^{*}\right)} s, \\
q(a) & :=\sum_{\left(s, s^{*}\right) \in \mathcal{C}\left(E \times E^{*}\right)} a_{\left(s, s^{*}\right)} s^{*}
\end{aligned}
$$

and

$$
r(a):=\sum_{\left(s, s^{*}\right) \in \mathcal{C}\left(E \times E^{*}\right)} a_{\left(s, s^{*}\right)}\left\langle s, s^{*}\right\rangle .
$$

We also define $\varphi: \mathcal{C}\left(E \times E^{*}\right) \times E^{*} \rightarrow \mathbb{R}$ by

$$
\varphi\left(a, x^{*}\right):=r(a)-\left\langle p(a), x^{*}\right\rangle .
$$

$\varphi$ is affine in each variable. We suppose now that $S: E \rightarrow 2^{E^{*}}$ is a monotone operator and $D(S) \neq \emptyset$, and we write

$$
\sigma_{S}:=\left\{a: a \in \mathcal{C}\left(E \times E^{*}\right), \quad a_{\left(s, s^{*}\right)}>0 \Longrightarrow\left(s, s^{*}\right) \in G(S)\right\} .
$$

We say that $a$ is a vertex of $\sigma_{S}$ if there exists $\left(y, y^{*}\right) \in G(S)$ such that

$$
a_{\left(s, s^{*}\right)}= \begin{cases}1 & \text { if }\left(s, s^{*}\right)=\left(y, y^{*}\right), \\ 0 & \text { otherwise. }\end{cases}
$$

The manipulations contained in Lemma 7 are part of the folklore of the theory of monotone operators.

Lemma 7. Let $S: E \rightarrow 2^{E^{*}}$ be a monotone operator with $D(S) \neq \emptyset$, and $a \in \sigma_{S}$. Then

$$
\varphi(a, q(a)) \geq 0 .
$$

Proof. Let $\left(s_{1}, s_{1}^{*}\right), \ldots,\left(s_{m}, s_{m}^{*}\right)$ be an enumeration of those elements $\left(s, s^{*}\right)$ of $G(S)$ for which $a_{\left(s, s^{*}\right)}>0$, and write $\alpha_{i}$ for $a_{\left(s_{i}, s_{i}^{*}\right)}$. Then

$$
\begin{aligned}
\varphi(a, q(a)) & =r(a)-\langle p(a), q(a)\rangle \\
& =\sum_{i} \alpha_{i}\left\langle s_{i}, s_{i}^{*}\right\rangle-\left\langle\sum_{i} \alpha_{i} s_{i}, \sum_{i} \alpha_{i} s_{i}^{*}\right\rangle \\
& =\sum_{i, j} \alpha_{i} \alpha_{j}\left\langle s_{i}, s_{i}^{*}\right\rangle-\sum_{i, j} \alpha_{i} \alpha_{j}\left\langle s_{i}, s_{j}^{*}\right\rangle \\
& =\sum_{i, j} \alpha_{i} \alpha_{j}\left\langle s_{i}, s_{i}^{*}-s_{j}^{*}\right\rangle \\
& =\sum_{i<j} \alpha_{i} \alpha_{j}\left\langle s_{i}, s_{i}^{*}-s_{j}^{*}\right\rangle+\sum_{j<i} \alpha_{i} \alpha_{j}\left\langle s_{i}, s_{i}^{*}-s_{j}^{*}\right\rangle \\
& =\sum_{i<j} \alpha_{i} \alpha_{j}\left\langle s_{i}, s_{i}^{*}-s_{j}^{*}\right\rangle+\sum_{i<j} \alpha_{i} \alpha_{j}\left\langle s_{j}, s_{j}^{*}-s_{i}^{*}\right\rangle \\
& =\sum_{i<j} \alpha_{i} \alpha_{j}\left\langle s_{i}-s_{j}, s_{i}^{*}-s_{j}^{*}\right\rangle \geq 0 .
\end{aligned}
$$

Definition 8. We define $\chi_{S}: E \rightarrow \mathbb{R} \cup\{\infty\}$ by

$$
\chi_{S}:=\sup _{a \in \sigma_{S}} \frac{q(a)-r(a)}{1+\|p(a)\|} .
$$


Since $\chi_{S}$ is the supremum of a family of continuous affine functions, $\chi_{S}$ is convex and lower semicontinuous. Lemma 9 shows that $\chi_{S}$ is also proper.

Lemma 9. Let $S: E \rightarrow 2^{E^{*}}$ be a monotone operator and $D(S) \neq \emptyset$. Then

$$
D(S) \subset \operatorname{co} D(S) \subset \operatorname{dom} \chi_{S} .
$$

Proof. Since dom $\chi_{S}$ is convex, it suffices to prove that

$$
D(S) \subset \operatorname{dom} \chi_{S} .
$$

To this end, let $w \in D(S)$. Pick $w^{*} \in S w$, and define $\beta:=\left\langle w, w^{*}\right\rangle \vee\left\|w^{*}\right\|$. If $\left(s, s^{*}\right) \in G(S)$, then, since $S$ is monotone,

$$
\left\langle w, s^{*}\right\rangle-\left\langle s, s^{*}\right\rangle=\left\langle w-s, s^{*}\right\rangle \leq\left\langle w-s, w^{*}\right\rangle=\left\langle w, w^{*}\right\rangle-\left\langle s, w^{*}\right\rangle .
$$

Thus, for all $a \in \sigma_{S}$ and $\left(s, s^{*}\right) \in G(S)$,

$$
\left\langle w, a_{\left(s, s^{*}\right)} s^{*}\right\rangle-a_{\left(s, s^{*}\right)}\left\langle s, s^{*}\right\rangle \leq a_{\left(s, s^{*}\right)}\left\langle w, w^{*}\right\rangle-\left\langle a_{\left(s, s^{*}\right)} s, w^{*}\right\rangle .
$$

Summing over $\left(s, s^{*}\right) \in G(S)$,

$$
\langle w, q(a)\rangle-r(a) \leq\left\langle w, w^{*}\right\rangle-\left\langle p(a), w^{*}\right\rangle \leq\left\langle w, w^{*}\right\rangle+\|p(a)\|\left\|w^{*}\right\| \leq \beta(1+\|p(a)\|) .
$$

Dividing by $1+\|p(a)\|$, and taking the supremum over $a \in \sigma_{S}$, we see that $\chi_{S}(w) \leq$ $\beta$, which implies that $w \in \operatorname{dom} \chi_{S}$. This completes the proof of (9.1), and hence that of Lemma 9.

If $T: E \rightarrow 2^{E^{*}}$, the inverse $T^{-1}: E^{*} \rightarrow 2^{E}$ of $T$ is defined by

$$
T^{-1} x^{*}:=\left\{x: x \in E, T x \ni x^{*}\right\} \quad\left(x^{*} \in E^{*}\right) .
$$

Lemma 10. Let $T: E \rightarrow 2^{E^{*}}$ be monotone and $D(T) \neq \emptyset$. Let $w \in E$ and $S=$ $\left(T^{-1}-w\right)^{-1}$. Then dom $\chi_{S}=\operatorname{dom} \chi_{T}-w$.

Proof. We note that $S x=T(x+w) \quad(x \in E)$. We shall prove that, for all $x \in E$,

$$
\frac{\chi_{S}(x)}{1+\|w\|} \leq \chi_{T}(x+w) \leq(1+\|w\|) \chi_{S}(x)
$$

from which the desired result follows. It fact, it suffices to prove the second inequality in (10.1) - the first inequality follows by replacing $w$ by $-w$ and interchanging the roles of $T$ and $S$. Let $a \in \sigma_{T}$, and define $b \in \sigma_{S}$ by $b_{\left(s, s^{*}\right)}=a_{\left(s+w, s^{*}\right)}$. We note that

$$
p(b)=p(a)-w, \quad q(a)=q(b) \quad \text { and } \quad r(a)-\langle w, q(a)\rangle=r(b) .
$$

Thus

$$
\langle x+w, q(a)\rangle-r(a)=\langle x, q(b)\rangle-r(b)
$$

from the definition of $\chi_{S}(x)$,

$$
\begin{aligned}
& \leq(1+\|p(b)\|) \chi_{S}(x) \\
& =(1+\|p(a)-w\|) \chi_{S}(x) \\
& \leq(1+\|p(a)\|+\|w\|) \chi_{S}(x) \\
& \leq(1+\|p(a)\|)(1+\|w\|) \chi_{S}(x) .
\end{aligned}
$$

The second inequality in (10.1) follows by dividing by $1+\|p(a)\|$, taking the supremum over $a$, and using the definition of $\chi_{T}(x+w)$. This completes the proof of Lemma 10. 
Lemma 11. Let $S: E \rightarrow 2^{E^{*}}$ be monotone and $D(S) \neq \emptyset$. Let $w^{*} \in E^{*}$ and $T=S-w^{*}$. Then $\operatorname{dom} \chi_{T}=\operatorname{dom} \chi_{S}$.

Proof. Let $x \in E$ and $\beta:=\left\|w^{*}\right\| \vee\|x\|\left\|w^{*}\right\|$. We shall prove that

$$
\chi_{T}(x)-\beta \leq \chi_{S}(x) \leq \chi_{T}(x)+\beta,
$$

from which the desired result follows. It fact, it suffices to prove the second inequality in (11.1) - the first inequality follows by replacing $w^{*}$ by $-w^{*}$ and interchanging the roles of $S$ and $T$. Let $a \in \sigma_{S}$, and define $b \in \sigma_{T}$ by $b_{\left(t, t^{*}\right)}=a_{\left(t, t^{*}+w^{*}\right)}$. We note that

$$
p(b)=p(a), \quad q(a)=q(b)+w^{*} \quad \text { and } \quad r(a)=r(b)+\left\langle p(b), w^{*}\right\rangle .
$$

Thus

$$
\begin{aligned}
\langle x, q(a)\rangle-r(a) & =\left\langle x, q(b)+w^{*}\right\rangle-r(b)-\left\langle p(b), w^{*}\right\rangle \\
& \leq\langle x, q(b)\rangle-r(b)+\|x\|\left\|w^{*}\right\|+\|p(b)\|\left\|w^{*}\right\| \\
& \leq\langle x, q(b)\rangle-r(b)+\beta+\beta\|p(b)\| \\
& =\langle x, q(b)\rangle-r(b)+\beta(1+\|p(b)\|)
\end{aligned}
$$

from the definition of $\chi_{T}(x)$,

$$
\begin{aligned}
& \leq(1+\|p(b)\|) \chi_{T}(x)+\beta(1+\|p(b)\|) \\
& =(1+\|p(a)\|)\left(\chi_{T}(x)+\beta\right) .
\end{aligned}
$$

The second inequality in (11.1) follows by dividing by $1+\|p(a)\|$, taking the supremum over $a$, and using the definition of $\chi_{S}(w)$.

Remark 12. The function $\chi_{S}$ was defined in [9], in which Lemmas 9 and 10 also appeared. Here, for the convenience of the reader, are the other main results proved in [9]. Let $x \in E$ and $A \subset E$. We write " $x \in \operatorname{sur} A$ " if, for each $w \in E \backslash\{0\}$, there exists $\delta>0$ such that $x+\delta w \in A$.

First, let $S: E \rightarrow 2^{E^{*}}$ be monotone and $D(S) \neq \emptyset$. Then:

(a) If $x \in \operatorname{sur}\left(\operatorname{dom} \chi_{S}\right)$, then there exist $\delta, Q>0$ such that

$$
\left(y, y^{*}\right) \in G(S) \text { and }\|y-x\|<\delta \quad \Longrightarrow \quad\left\|y^{*}\right\| \leq Q .
$$

(b) If $S: E \rightarrow 2^{E^{*}}$ is maximal monotone, then

$$
\text { int } \begin{aligned}
D(S) & =\operatorname{int}(\operatorname{co} D(S))=\operatorname{int}\left(\operatorname{dom} \chi_{S}\right) \\
& =\operatorname{sur} D(S)=\operatorname{sur}(\operatorname{co} D(S))=\operatorname{sur}\left(\operatorname{dom} \chi_{S}\right) .
\end{aligned}
$$

(c) If $S: E \rightarrow 2^{E^{*}}$ is maximal monotone and $\operatorname{sur}\left(\operatorname{dom} \chi_{S}\right) \neq \emptyset$, then

$$
\begin{aligned}
\overline{D(S)} & =\overline{\operatorname{co} D(S)}=\overline{\operatorname{dom} \chi_{S}} \\
& =\overline{\operatorname{int} D(S)}=\overline{\operatorname{int}(\operatorname{co} D(S))}=\overline{\operatorname{int}\left(\operatorname{dom} \chi_{S}\right)} \\
& =\overline{\operatorname{sur} D(S)}=\overline{\operatorname{sur}(\operatorname{co} D(S))}=\overline{\operatorname{sur}\left(\operatorname{dom} \chi_{S}\right)} .
\end{aligned}
$$

The statement " $x \in \operatorname{sur} A$ " is related to $x$ being an "absorbing point" of $A$ (see [14], Definition 2.27(b), p. 28), but differs in that we do not require that $x \in A$. (a) strengthens the local boundedness result of Borwein-Fitzpatrick (see [6]). (b) and (c) make more explicit the result proved by Rockafellar in [17], Theorem 1, p. 398 (see also [15], Theorem 1.9, p. 6) that if $S$ is maximal monotone and 
$\operatorname{int}(\operatorname{co} D(S)) \neq \emptyset$, then int $D(S)$ and $\overline{D(S)}$ are both convex. (b) also answers in the affirmative a question raised by Phelps (see [14], p. 29 and [15], p. 8).

\section{The “DOM-DOM” CONSTRAINT QUALIFICATION}

Our next series of results concerns pairs of monotone operators that satisfy the dom-dom constraint qualification (0.4).

Lemma 13. Let $E$ be reflexive, $S_{1}, S_{2}: E \rightarrow 2^{E^{*}}$ be monotone operators and dom $\chi_{S_{1}}-\operatorname{dom} \chi_{S_{2}}$ be absorbing. Write $A:=\sigma_{S_{1}} \times \sigma_{S_{2}}$, and define the function $f: A \times\left(E^{*} \times E^{*} \times E\right) \rightarrow \mathbb{R}$ by the formula

$$
\begin{aligned}
& f\left(\left(a_{1}, a_{2}\right),\left(x_{1}^{*}, x_{2}^{*}, z\right)\right) \\
& \quad:=2 \varphi\left(a_{1}, x_{1}^{*}\right)+2 \varphi\left(a_{2}, x_{2}^{*}\right)-2\left\langle z, q\left(a_{1}\right)+q\left(a_{2}\right)\right\rangle-\|z\|^{2}-\left\|x_{1}^{*}+x_{2}^{*}\right\|^{2} .
\end{aligned}
$$

Then there exists $M>0$ with the following property: for all $\left(a_{1}, a_{2}\right) \in A$, there exists $\left(x_{1}^{*}, x_{2}^{*}, z\right) \in E^{*} \times E^{*} \times E$ such that

$$
\left\|x_{1}^{*}\right\| \leq M, \quad\left\|x_{2}^{*}\right\| \leq M, \quad\|z\| \leq 2 M \quad \text { and } \quad f\left(\left(a_{1}, a_{2}\right),\left(x_{1}^{*}, x_{2}^{*}, z\right)\right) \geq 0 .
$$

Proof. To simplify the expressions in what follows, we write $\chi_{i}$ instead of $\chi_{S_{i}}$. From Corollary 4 , there exist $n \geq 1$ and $\eta \in(0,1]$ such that

$$
w \in E \text { and }\|w\| \leq \eta \quad \Longrightarrow \quad w \in E\left\{\chi_{1} \vee\|\| \leq n\right\}-E\left\{\chi_{2} \vee\|\| \leq n\right\} .
$$

We write $M:=5 n^{2} / \eta$, and we shall show that $M$ has the required property. So let $\left(a_{1}, a_{2}\right) \in A$. We start off by proving that there exists $\left(x_{1}^{*}, x_{2}^{*}, \nu\right) \in E^{*} \times E^{*} \times \mathbb{R}$ such that

$$
\left\{\begin{array}{c}
\left\|x_{1}^{*}\right\| \leq M, \quad\left\|x_{2}^{*}\right\| \leq M, \quad \nu \in[0,2 M] \\
\text { and } \\
2 \varphi\left(a_{1}, x_{1}^{*}\right)+2 \varphi\left(a_{2}, x_{2}^{*}\right)+2 \nu\left\|q\left(a_{1}\right)+q\left(a_{2}\right)\right\|-\nu^{2}-\left\|x_{1}^{*}+x_{2}^{*}\right\|^{2} \geq 0 .
\end{array}\right.
$$

We first consider the case that $\left\|q\left(a_{1}\right)\right\| \vee\left\|q\left(a_{2}\right)\right\| \leq M$. Here we write $\left(x_{1}^{*}, x_{2}^{*}\right)=$ $\left(q\left(a_{1}\right), q\left(a_{2}\right)\right)$ and $\nu:=\left\|q\left(a_{1}\right)+q\left(a_{2}\right)\right\|$, and (13.3) is immediate from Lemma 7 . We now consider the other alternative, namely that $\left\|q\left(a_{1}\right)\right\| \vee\left\|q\left(a_{2}\right)\right\|>M$, and we shall prove that

(13.3) is satisfied with $\left\|x_{i}^{*}\right\| \leq n$ and $\nu=n$

We fix $j \in\{1,2\}$ such that $\left\|q\left(a_{j}\right)\right\|>M$, choose an element $v$ of $E$ such that

$$
\|v\| \leq \eta \quad \text { and } \quad\left\langle v, q\left(a_{j}\right)\right\rangle \geq M \eta=5 n^{2},
$$

and write $k$ for the element of $\{1,2\}$ different from $j$. From (13.2) applied to $w:= \pm v$ (depending on whether $j=1$ or 2), there exist $x, y \in E$ such that

$$
\chi_{j}(x) \leq n, \quad \chi_{k}(y) \leq n, \quad\|y\| \leq n \quad \text { and } \quad x-y=v .
$$

From the Hahn-Banach theorem, there exist $x_{1}^{*}, x_{2}^{*} \in E^{*}$ such that, for $i=1,2$,

$$
\left\|x_{i}^{*}\right\| \leq n, \quad \text { and } \quad\left\langle p\left(a_{i}\right), x_{i}^{*}\right\rangle=-n\left\|p\left(a_{i}\right)\right\| .
$$

Since $M \geq n,\left\|x_{1}^{*}\right\| \leq M$ and $\left\|x_{2}^{*}\right\| \leq M$ as required. Further, since $\chi_{j}(x) \leq n$ and $x=v+y$,

$$
\begin{aligned}
\varphi\left(a_{j}, x_{j}^{*}\right) & :=r\left(a_{j}\right)-\left\langle p\left(a_{j}\right), x_{j}^{*}\right\rangle \\
& =r\left(a_{j}\right)+n\left\|p\left(a_{j}\right)\right\| \geq\left\langle x, q\left(a_{j}\right)\right\rangle-n \\
& =\left\langle v+y, q\left(a_{j}\right)\right\rangle-n=\left\langle v, q\left(a_{j}\right)\right\rangle+\left\langle y, q\left(a_{j}\right)\right\rangle-n .
\end{aligned}
$$


Thus, from (13.5),

$$
\varphi\left(a_{j}, x_{j}^{*}\right) \geq 5 n^{2}+\left\langle y, q\left(a_{j}\right)\right\rangle-n \geq 4 n^{2}+\left\langle y, q\left(a_{j}\right)\right\rangle .
$$

Since $\chi_{k}(y) \leq n$,

$$
\varphi\left(a_{k}, x_{k}^{*}\right):=r\left(a_{k}\right)-\left\langle p\left(a_{k}\right), x_{k}^{*}\right\rangle=r\left(a_{k}\right)+n\left\|p\left(a_{k}\right)\right\| \geq\left\langle y, q\left(a_{k}\right)\right\rangle-n .
$$

We now add (13.6) and (13.7). Since $\|y\| \leq n$, we derive

$$
\varphi\left(a_{1}, x_{1}^{*}\right)+\varphi\left(a_{2}, x_{2}^{*}\right) \geq 4 n^{2}+\left\langle y, q\left(a_{1}\right)+q\left(a_{2}\right)\right\rangle-n \geq 3 n^{2}-n\left\|q\left(a_{1}\right)+q\left(a_{2}\right)\right\| .
$$

Thus

$$
\begin{gathered}
2 \varphi\left(a_{1}, x_{1}^{*}\right)+2 \varphi\left(a_{2}, x_{2}^{*}\right)+2 n\left\|q\left(a_{1}\right)+q\left(a_{2}\right)\right\|-n^{2}-\left\|x_{1}^{*}+x_{2}^{*}\right\|^{2} \\
\geq 6 n^{2}-n^{2}-\left\|x_{1}^{*}+x_{2}^{*}\right\|^{2},
\end{gathered}
$$

since $\left\|x_{1}^{*}\right\| \leq n$ and $\left\|x_{2}^{*}\right\| \leq n$,

$$
\geq 5 n^{2}-(2 n)^{2}>0 \text {. }
$$

Thus (13.4) is satisfied. This completes the proof of (13.3). Since $E$ is reflexive, we can choose $z \in E$ such that $\|z\| \leq \nu$ and

$$
\left\langle z, q\left(a_{1}\right)+q\left(a_{2}\right)\right\rangle=-\nu\left\|q\left(a_{1}\right)+q\left(a_{2}\right)\right\|,
$$

from which (13.1) is an immediate consequence.

The minimax theorem below follows from a result of Fan (see [11]). (See also [13] and [19] for simple generalizations of Fan's result.)

Theorem 14. Let $A$ be a nonempty convex subset of a vector space, and $B$ be a nonempty compact convex subset of a topological vector space. Let $h: A \times B \rightarrow \mathbb{R}$ be convex on $A$, and concave and upper semicontinuous on $B$. Then

$$
\inf _{A} \max _{B} h=\max _{B} \inf _{A} h .
$$

Lemma 15. Let $E$ be reflexive, $S_{1}, S_{2}: E \rightarrow 2^{E^{*}}$ be monotone operators and dom $\chi_{S_{1}}-\operatorname{dom} \chi_{S_{2}}$ be absorbing. Then there exists $\left(x_{1}^{*}, x_{2}^{*}, z\right) \in E^{*} \times E^{*} \times E$ such that,

$$
\text { for all }\left(a_{1}, a_{2}\right) \in A, \quad f\left(\left(a_{1}, a_{2}\right),\left(x_{1}^{*}, x_{2}^{*}, z\right)\right) \geq 0 .
$$

Furthermore, for all $\left(s_{1}, s_{1}^{*}\right) \in G\left(S_{1}\right)$ and $\left(s_{2}, s_{2}^{*}\right) \in G\left(S_{2}\right)$,

$$
2 \sum_{i=1}^{2}\left[\left\langle s_{i}, s_{i}^{*}\right\rangle-\left\langle s_{i}, x_{i}^{*}\right\rangle-\left\langle z, s_{i}^{*}\right\rangle\right]-\|z\|^{2}-\left\|x_{1}^{*}+x_{2}^{*}\right\|^{2} \geq 0 .
$$

Proof. We choose $M$ as in Lemma 13, and write

$$
B:=\left\{\left(x_{1}^{*}, x_{2}^{*}, z\right):\left\|x_{1}^{*}\right\| \leq M,\left\|x_{2}^{*}\right\| \leq M,\|z\| \leq 2 M\right\} \subset E^{*} \times E^{*} \times E .
$$

It follows from Lemma 13 that

$$
\inf _{A} \max _{B} f \geq 0 .
$$

$A$ and $B$ are convex. We give $E$ and $E^{*}$ their weak topologies, and it then follows from the Banach-Alaoglu theorem that $B$ is compact. Further, $f$ is affine on $A$, and concave and upper semicontinuous on $B$. Thus, from Theorem 14,

$$
\max _{B} \inf _{A} f \geq 0
$$


which gives (15.1). (15.2) follows from (15.1) by allowing $a_{i}$ to run through the vertices of $\sigma_{S_{i}}$. This completes the proof of Lemma 15 .

\section{MAXIMAL MONOTONE OPERATORS ON REFLEXIVE SPACES}

Lemma 16 contains the essence of maximal monotonicity. (a) follows by taking $\left(s, s^{*}\right):=\left(z, z^{*}\right),(\mathrm{b})$ is immediate from the definition of maximal monotonicity, and (c) follows from (a) and (b).

Lemma 16. Let $S: E \rightarrow 2^{E^{*}}$ be maximal monotone.

(a) If $\left(z, z^{*}\right) \in G(S)$, then $\inf _{\left(s, s^{*}\right) \in G(S)}\left\langle s-z, s^{*}-z^{*}\right\rangle \leq 0$.

(b) If $\left(z, z^{*}\right) \in E \times E^{*} \backslash G(S)$, then $\inf _{\left(s, s^{*}\right) \in G(S)}\left\langle s-z, s^{*}-z^{*}\right\rangle<0$.

(c) For all $\left(z, z^{*}\right) \in E \times E^{*}, \inf _{\left(s, s^{*}\right) \in G(S)}\left\langle s-z, s^{*}-z^{*}\right\rangle \leq 0$.

Lemma 17. Let $E$ be reflexive, $S_{1}, S_{2}: E \rightarrow 2^{E^{*}}$ be maximal monotone operators and dom $\chi_{S_{1}}-\operatorname{dom} \chi_{S_{2}}$ be absorbing. Then there exists $\left(z, z^{*}\right) \in G\left(S_{1}+S_{2}\right)$ such that

$$
\|z\|^{2}+\left\|z^{*}\right\|^{2}+2\left\langle z, z^{*}\right\rangle=0
$$

Proof. From Lemma 15, there exists $\left(x_{1}^{*}, x_{2}^{*}, z\right) \in E^{*} \times E^{*} \times E$ such that, for all $\left(s_{1}, s_{1}^{*}\right) \in G\left(S_{1}\right)$ and $\left(s_{2}, s_{2}^{*}\right) \in G\left(S_{2}\right)$,

$$
2 \sum_{i=1}^{2}\left[\left\langle s_{i}, s_{i}^{*}\right\rangle-\left\langle s_{i}, x_{i}^{*}\right\rangle-\left\langle z, s_{i}^{*}\right\rangle\right] \geq\|z\|^{2}+\left\|x_{1}^{*}+x_{2}^{*}\right\|^{2},
$$

from which

$$
2 \sum_{i=1}^{2}\left[\left\langle s_{i}, s_{i}^{*}\right\rangle-\left\langle s_{i}, x_{i}^{*}\right\rangle-\left\langle z, s_{i}^{*}\right\rangle+\left\langle z, x_{i}^{*}\right\rangle\right] \geq\|z\|^{2}+\left\|x_{1}^{*}+x_{2}^{*}\right\|^{2}+2\left\langle z, x_{1}^{*}+x_{2}^{*}\right\rangle .
$$

Putting $z^{*}:=x_{1}^{*}+x_{2}^{*}$, this can be rewritten

$$
2 \sum_{i=1}^{2}\left\langle s_{i}-z, s_{i}^{*}-x_{i}^{*}\right\rangle \geq\|z\|^{2}+\left\|z^{*}\right\|^{2}+2\left\langle z, z^{*}\right\rangle .
$$

Taking the infimum over all $\left(s_{1}, s_{1}^{*}\right) \in G\left(S_{1}\right)$ and $\left(s_{2}, s_{2}^{*}\right) \in G\left(S_{2}\right)$,

$$
2 \sum_{i=1}^{2} \inf _{\left(s_{i}, s_{i}^{*}\right) \in G\left(S_{i}\right)}\left\langle s_{i}-z, s_{i}^{*}-x_{i}^{*}\right\rangle \geq\|z\|^{2}+\left\|z^{*}\right\|^{2}+2\left\langle z, z^{*}\right\rangle .
$$

Since $\|z\|^{2}+\left\|z^{*}\right\|^{2}+2\left\langle z, z^{*}\right\rangle \geq\|z\|^{2}+\left\|z^{*}\right\|^{2}-2\|z\|\left\|z^{*}\right\| \geq 0$, it follows from Lemma 16 (c) that (17.1) is satisfied and, for $i=1,2$,

$$
\inf _{\left(s_{i}, s_{i}^{*}\right) \in G\left(S_{i}\right)}\left\langle s_{i}-z, s_{i}^{*}-x_{i}^{*}\right\rangle=0 .
$$

We now deduce from Lemma 16 (b) that $\left(z, x_{i}^{*}\right) \in G\left(S_{i}\right)$. Consequently,

$$
\left(z, z^{*}\right)=\left(z, x_{1}^{*}+x_{2}^{*}\right) \in G\left(S_{1}+S_{2}\right)
$$

This completes the proof of Lemma 17.

Lemma 18. Let $E$ be reflexive, $T_{1}, T_{2}: E \rightarrow 2^{E^{*}}$ be maximal monotone operators and dom $\chi_{T_{1}}-\operatorname{dom} \chi_{T_{2}}$ be absorbing. Suppose also that $w \in E$. Then there exists $\left(\zeta, z^{*}\right) \in G\left(T_{1}+T_{2}\right)$ such that

$$
\|\zeta-w\|^{2}+\left\|z^{*}\right\|^{2}+2\left\langle\zeta-w, z^{*}\right\rangle=0
$$


Proof. Let $S_{i}:=\left(T_{i}^{-1}-w\right)^{-1}$. From Lemma 10, dom $\chi_{S_{1}}-\operatorname{dom} \chi_{S_{2}}$ is absorbing. The result follows from Lemma 17 , with $\zeta:=z+w$.

Theorem 19. Let $E$ be reflexive, $S_{1}, S_{2}: E \rightarrow 2^{E^{*}}$ be maximal monotone operators and dom $\chi_{S_{1}}-\operatorname{dom} \chi_{S_{2}}$ be absorbing. Suppose also that $w \in E$ and $w^{*} \in E^{*}$. Then there exists $\left(\zeta, \zeta^{*}\right) \in G\left(S_{1}+S_{2}\right)$ such that

$$
\|\zeta-w\|^{2}+\left\|\zeta^{*}-w^{*}\right\|^{2}+2\left\langle\zeta-w, \zeta^{*}-w^{*}\right\rangle=0 .
$$

Proof. Let $T_{i}:=S_{i}-w^{*} / 2$. From Lemma 11, dom $\chi_{T_{1}}-\operatorname{dom} \chi_{T_{2}}$ is absorbing. The result follows from Lemma 18 , with $\zeta^{*}:=z^{*}+w^{*}$.

Corollary 20. Let $E$ be reflexive, $S_{1}, S_{2}: E \rightarrow 2^{E^{*}}$ be maximal monotone operators and dom $\chi_{S_{1}}-\operatorname{dom} \chi_{S_{2}}$ be absorbing. Then $S_{1}+S_{2}$ is maximal monotone.

Proof. Suppose that $\left(w, w^{*}\right) \in E \times E^{*}$ and,

$$
\text { for all }\left(z, z^{*}\right) \in G\left(S_{1}+S_{2}\right), \quad\left\langle z-w, z^{*}-w^{*}\right\rangle \geq 0 .
$$

Choose $\left(\zeta, \zeta^{*}\right) \in G\left(S_{1}+S_{2}\right)$ as in (19.1). From (20.1) with $\left(z, z^{*}\right):=\left(\zeta, \zeta^{*}\right)$,

$$
\|\zeta-w\|^{2}+\left\|\zeta^{*}-w^{*}\right\|^{2} \leq 0
$$

hence $\zeta=w$ and $\zeta^{*}=w^{*}$. Thus $\left(w, w^{*}\right)=\left(\zeta, \zeta^{*}\right) \in G\left(S_{1}+S_{2}\right)$. This completes the proof of Corollary 20.

Remark 21. We have not yet explicitly introduced the duality mapping $J$, though it has been implicit in some of the above results. For instance, (17.1) is equivalent to the statement that $-z^{*} \in J z$, so the conclusion of Lemma 17 is that $R\left(S_{1}+S_{2}+J\right) \ni$ 0 . If $w \in E$, define $J_{w}(x):=J(x-w) \quad(x \in E)$. Then (19.1) is equivalent to the statement that $w^{*}-\zeta^{*} \in J_{w}(\zeta)$, so the conclusion of Theorem 19 is that, for all $w \in E, S_{1}+S_{2}+J_{w}$ is surjective. The simple bootstrapping that we have done in going from Lemma 17 to Theorem 19 (i.e., proving the surjectivity of $S_{1}+S_{2}+J_{w}$ rather than that of $\left.S_{1}+S_{2}+J\right)$ is what makes it unnecessary to use a renorming theorem.

We now discuss the comment made in the introduction that the function $f$ defined in Lemma 13 is not pulled out of a hat. Suppose we know that $S_{1}+S_{2}$ is maximal monotone. As we have observed in the introduction, $S_{1}+S_{2}+J$ is surjective; consequently there exists $\left(z, z^{*}\right) \in G\left(S_{1}+S_{2}\right)$ such that (17.1) is satisfied. Let $\left(z, x_{1}^{*}\right) \in G\left(S_{1}\right),\left(z, x_{2}^{*}\right) \in G\left(S_{2}\right)$ and $x_{1}^{*}+x_{2}^{*}=z^{*}$. Then, for all $\left(s_{1}, s_{1}^{*}\right) \in G\left(S_{1}\right)$ and $\left(s_{2}, s_{2}^{*}\right) \in G\left(S_{2}\right),(17.2)$ is satisfied, and so (15.2) is also satisfied. From convexity, (15.1) is also satisfied, from which, for all $\left(a_{1}, a_{2}\right) \in A$,

$$
2 \varphi\left(a_{1}, x_{1}^{*}\right)+2 \varphi\left(a_{2}, x_{2}^{*}\right)+2\|z\|\left\|q\left(a_{1}\right)+q\left(a_{2}\right)\right\|-\|z\|^{2}-\left\|x_{1}^{*}+x_{2}^{*}\right\|^{2} \geq 0 .
$$

Let $M \geq\left\|x_{1}^{*}\right\|, M \geq\left\|x_{2}^{*}\right\|$ and $M \geq\|z\| / 2$. Then (13.3) is satisfied with $\nu:=\|z\|$.

Put another way, there is no real loss between (13.3) and Corollary 20. The problem is, of course, to find a value of $M$ that makes it possible to establish (13.3).

\section{The $\chi$ CONSTRAINT QUALIFICATION}

Corollary 20 is enough to establish the sum theorem under the assumptions (0.1)(0.3). In order to deal with (0.5)-(0.7), we introduce a new constraint qualification. 
Definition 22. Let $S_{1}, S_{2}: E \rightarrow 2^{E^{*}}$ be monotone operators. We say that $S_{1}, S_{2}$ satisfy the $\chi$ constraint qualification if there exist $w \in E$ and a closed subspace $F$ of $E$ such that $D\left(S_{1}\right) \cup D\left(S_{2}\right) \subset w+F$ and

$$
\bigcup_{\lambda>0} \lambda\left[(w+F) \cap \operatorname{dom} \chi_{S_{1}}-(w+F) \cap \operatorname{dom} \chi_{S_{2}}\right]=F .
$$

It is clear from Lemma 23 below that if any of (0.1)-(0.6) is satisfied then $S_{1}, S_{2}$ satisfy the $\chi$ constraint qualification.

Lemma 23. Let $S_{1}, S_{2}: E \rightarrow 2^{E^{*}}$ be monotone operators and

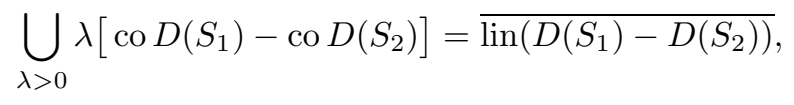

i.e., (0.7) is satisfied. Then $S_{1}, S_{2}$ satisfy the $\chi$ constraint qualification.

Proof. Let $F:=\overline{\operatorname{lin}\left(D\left(S_{1}\right)-D\left(S_{2}\right)\right)}$. Since $0 \in F, 0 \in \operatorname{co} D\left(S_{1}\right)-\operatorname{co} D\left(S_{2}\right)$; hence there exists $w \in \operatorname{co} D\left(S_{1}\right) \cap \operatorname{co} D\left(S_{2}\right)$. But then

$$
D\left(S_{1}\right)-w \subset \operatorname{co} D\left(S_{1}\right)-\operatorname{co} D\left(S_{2}\right)=\operatorname{co}\left(D\left(S_{1}\right)-D\left(S_{2}\right)\right) \subset F
$$

and

$$
D\left(S_{2}\right)-w \subset \operatorname{co} D\left(S_{2}\right)-\operatorname{co} D\left(S_{1}\right)=\operatorname{co}\left(D\left(S_{2}\right)-D\left(S_{1}\right)\right) \subset F ;
$$

consequently $D\left(S_{1}\right) \cup D\left(S_{2}\right) \subset w+F$. It then follows from Lemma 9 that, for $i=1,2$, co $D\left(S_{i}\right) \subset(w+F) \cap \operatorname{dom} \chi_{S_{i}}$, which gives the desired result.

Using an argument similar to that employed in Lemma 23, one can prove that if

$$
\bigcup_{\lambda>0} \lambda\left[\operatorname{dom} \chi_{S_{1}}-\operatorname{dom} \chi_{S_{2}}\right]=\overline{\operatorname{lin}\left(\operatorname{dom} \chi_{S_{1}}-\operatorname{dom} \chi_{S_{2}}\right)}
$$

then $S_{1}, S_{2}$ satisfy the $\chi$ constraint qualification. However, our knowledge of the relationship between co $D(S)$ and dom $\chi_{S}$ is far from complete at the moment, and we do not know if (23.1) is, in fact, different from (0.7). (Compare Remark 12 (c).)

In order to bootstrap Corollary 20 into Theorem 26, we now digress a little and discuss the relationship between maximal monotone operators and subspaces.

Definition 24. Let $F$ be a subspace of a normed space $E$ and $T: E \rightarrow 2^{E^{*}}$. We say that $T$ is $F$-saturated if

$$
\left(u, u^{*}\right) \in G(T), v^{*} \in E^{*} \text { and }\left.v^{*}\right|_{F}=\left.u^{*}\right|_{F} \quad \Longrightarrow \quad\left(u, v^{*}\right) \in G(T) .
$$

Lemma 25. Let $F$ be a closed subspace of a normed space $E, T: E \rightarrow 2^{E^{*}}$ be monotone and $D(T) \subset F$. Define $\left.T\right|_{F}: F \rightarrow 2^{F^{*}}$ by $\left(\left.T\right|_{F}\right) x:=\left\{\left.x^{*}\right|_{F}: x^{*} \in T x\right\}$.

(a) $\left.T\right|_{F}$ is monotone.

(b) $\chi_{\left(\left.T\right|_{F}\right)}=\left.\left(\chi_{T}\right)\right|_{F}-$ hence dom $\chi_{\left(\left.T\right|_{F}\right)}=F \cap \operatorname{dom} \chi_{T}$.

(c) $T$ is maximal monotone $\Longleftrightarrow T$ is $F$-saturated and $\left.T\right|_{F}$ is maximal monotone.

Proof. We leave to the reader the proofs of (a) and (b), which are fairly straightforward.

(c) $\Longrightarrow$ Suppose that $T$ is maximal monotone. We first prove that $T$ is $F$ saturated. So let $\left(u, u^{*}\right) \in G(T), v^{*} \in E^{*}$ and $\left.v^{*}\right|_{F}=\left.u^{*}\right|_{F}$. From the monotonicity of $T$,

$$
\text { for all }\left(t, t^{*}\right) \in G(T), \quad\left\langle t-u, t^{*}-u^{*}\right\rangle \geq 0 \text {. }
$$


However, $t-u \in F$ and $\left.v^{*}\right|_{F}=\left.u^{*}\right|_{F} ;$ consequently,

$$
\text { for all }\left(t, t^{*}\right) \in G(T), \quad\left\langle t-u, t^{*}-v^{*}\right\rangle \geq 0 .
$$

From the maximal monotonicity of $T,\left(u, v^{*}\right) \in G(T)$. This completes the proof that $T$ is $F$-saturated. We next prove that $\left.T\right|_{F}$ is maximal monotone. Let $\left(z, z^{*}\right) \in$ $F \times F^{*}$ and

$$
\text { for all }\left(t, s^{*}\right) \in G\left(\left.T\right|_{F}\right), \quad\left\langle t-z, s^{*}-z^{*}\right\rangle \geq 0 \text {. }
$$

It follows that

$$
\text { for all }\left(t, t^{*}\right) \in G(T), \quad\left\langle t-z,\left.t^{*}\right|_{F}-z^{*}\right\rangle \geq 0 \text {. }
$$

From the Hahn-Banach theorem, there exists $y^{*} \in E^{*}$ such that $\left.y^{*}\right|_{F}=z^{*}$. We then have

$$
\text { for all }\left(t, t^{*}\right) \in G(T), \quad\left\langle t-z,\left.t^{*}\right|_{F}-\left.y^{*}\right|_{F}\right\rangle \geq 0,
$$

or equivalently, since $t-z \in F$,

$$
\text { for all }\left(t, t^{*}\right) \in G(T), \quad\left\langle t-z, t^{*}-y^{*}\right\rangle \geq 0 .
$$

From the maximal monotonicity of $T,\left(z, y^{*}\right) \in G(T)$; hence $\left(z, z^{*}\right)=\left(z,\left.y^{*}\right|_{F}\right) \in$ $G\left(\left.T\right|_{F}\right)$. Thus $\left.T\right|_{F}$ is maximal monotone, as required.

$(\mathrm{c})(\Longleftarrow) \quad$ We now assume that $T$ is $F$-saturated and $\left.T\right|_{F}$ is maximal monotone, and prove that $T$ is maximal monotone. Suppose that $\left(z, z^{*}\right) \in E \times E^{*}$ and

$$
\text { for all }\left(t, t^{*}\right) \in G(T), \quad\left\langle t-z, t^{*}-z^{*}\right\rangle \geq 0 .
$$

We first prove that

$$
y^{*} \in E^{*} \text { and }\left.y^{*}\right|_{F}=0 \quad \Longrightarrow \quad\left\langle z, y^{*}\right\rangle=0 .
$$

Let $y^{*} \in E^{*}$ and $\left.y^{*}\right|_{F}=0$, and fix $\left(t, t^{*}\right) \in G(T)$. We note, for future reference, that

$$
t \in F \text {. }
$$

Let $\lambda$ be an arbitrary real number. Since $T$ is $F$-saturated, $\left(t, t^{*}+\lambda y^{*}\right) \in G(T)$. Thus, from $(25.1),\left\langle t-z, t^{*}+\lambda y^{*}-z^{*}\right\rangle \geq 0$. We derive from this that

$$
\lambda\left\langle z, y^{*}\right\rangle=\lambda\left\langle z-t, y^{*}\right\rangle \leq\left\langle t-z, t^{*}-z^{*}\right\rangle .
$$

Since this holds for all $\lambda \in \mathbb{R},\left\langle z, y^{*}\right\rangle=0$, which completes the proof of (25.2). Since $F$ is closed, it now follows from (25.2) and the separation theorem that

$$
z \in F \text {. }
$$

Now let $\left(t, s^{*}\right)$ be an arbitrary element of $G\left(\left.T\right|_{F}\right)$. (25.3) is again satisfied. From the Hahn-Banach theorem, there exists $t^{*} \in E^{*}$ such that $\left.t^{*}\right|_{F}=s^{*}$. Since $T$ is $F$-saturated, $\left(t, t^{*}\right) \in G(T)$ so, from $(25.1),\left\langle t-z, t^{*}-z^{*}\right\rangle \geq 0$. We now derive from (25.4) and (25.3) that $\left\langle t-z,\left.t^{*}\right|_{F}-\left.z^{*}\right|_{F}\right\rangle \geq 0$; that is to say, $\left\langle t-z, s^{*}-\left.z^{*}\right|_{F}\right\rangle \geq 0$. Since this holds for all $\left(t, s^{*}\right) \in G\left(\left.T\right|_{F}\right)$ and $\left.T\right|_{F}$ is maximal monotone, $\left(z,\left.z^{*}\right|_{F}\right) \in$ $G\left(\left.T\right|_{F}\right)$. Finally, using the fact that $T$ is $F$-saturated, we obtain that $\left(z, z^{*}\right) \in G(T)$. This completes the proof that $T$ is maximal monotone.

Theorem 26. Let $E$ be reflexive and $S_{1}, S_{2}: E \rightarrow 2^{E^{*}}$ be maximal monotone operators satisfying the $\chi$ constraint qualification. Then $S_{1}+S_{2}$ is maximal monotone. 
Proof. Let $w$ and $F$ be as in Definition 22 and $T_{i}:=\left(S_{i}^{-1}-w\right)^{-1}$. Clearly, $T_{1}$ and $T_{2}$ are maximal monotone. From Lemma 10, $D\left(T_{1}\right) \cup D\left(T_{2}\right) \subset F$ and

$$
\bigcup_{\lambda>0} \lambda\left[F \cap \operatorname{dom} \chi_{T_{1}}-F \cap \operatorname{dom} \chi_{T_{2}}\right]=F
$$

From Lemma $25(\mathrm{c})(\Longrightarrow),\left.T_{1}\right|_{F}$ and $\left.T_{2}\right|_{F}$ are maximal monotone and, from Lemma 25 (b),

$$
\bigcup_{\lambda>0} \lambda\left[\operatorname{dom} \chi_{\left(\left.T_{1}\right|_{F}\right)}-\operatorname{dom} \chi_{\left(\left.T_{2}\right|_{F}\right)}\right]=F .
$$

Thus, from Corollary 20, $\left.\left(T_{1}+T_{2}\right)\right|_{F}=\left.T_{1}\right|_{F}+\left.T_{2}\right|_{F}$ is maximal monotone. From Lemma $25(\mathrm{c})(\Longrightarrow)$ again, $T_{1}$ and $T_{2}$ are $F$-saturated, hence $T_{1}+T_{2}$ is $F$-saturated. We now deduce from Lemma $25(\mathrm{c})(\Longleftarrow)$ that $T_{1}+T_{2}$ is maximal monotone and hence, from Lemma 10 again, that $S_{1}+S_{2}$ is maximal monotone too.

Corollary 27 is immediate from Theorem 26 and induction.

Corollary 27. Let $E$ be reflexive, $m \geq 2$ and $S_{1}, \ldots, S_{m}: E \rightarrow 2^{E^{*}}$ be maximal monotone operators such that, for all $k=2, \ldots, m, S_{1}+\cdots+S_{k-1}$ and $S_{k}$ satisfy the $\chi$ constraint qualification. Then $S_{1}+\cdots+S_{m}$ is maximal monotone.

Remark 28. It follows from Lemma 23 that $S_{1}+\cdots+S_{k-1}$ and $S_{k}$ satisfy the $\chi$ constraint qualification provided that

$$
\bigcup_{\lambda>0} \lambda\left[\operatorname{co} D\left(S_{1}+\cdots+S_{k-1}\right)-\operatorname{co} D\left(S_{k}\right)\right]=\overline{\operatorname{lin}\left(D\left(S_{1}+\cdots+S_{k-1}\right)-D\left(S_{k}\right)\right)} .
$$

If $C_{k}=D\left(S_{1}\right) \cap \cdots \cap D\left(S_{k-1}\right)-D\left(S_{k}\right)$, then (28.1) can be rewritten:

$$
\bigcup_{\lambda>0} \lambda\left[\operatorname{co} C_{k}\right]=\overline{\operatorname{lin} C_{k}} \text {. }
$$

Thus Corollary 27 generalizes [10], Corollary 4.2, in which the assumptions imply that

$$
\text { co } C_{k} \text { is a neighborhood of } 0 \text { relative to } \overline{\operatorname{lin} C_{k}} \text {. }
$$

\section{A Fenchel Duality Theorem for two or more Functions}

We suppose throughout this final section that $E$ and $F$ are Banach spaces. If $g: E \rightarrow \mathbb{R} \cup\{\infty\}$ is a proper convex, lower semicontinuous function, the conjugate function $g^{*}: E^{*} \rightarrow \mathbb{R} \cup\{\infty\}$ is defined by the formula $g^{*}\left(x^{*}\right):=\sup _{E}\left(x^{*}-g\right)$. $g^{*}$ is proper, convex and lower semicontinuous. The Fenchel Duality Theorem says that, under the appropriate constraint qualification, the conjugate of the sum of proper convex, lower semicontinuous functions is the exact episum of the conjugates of the functions. After some simple transformations, this problem can be reduced to finding a formula for the infimum of the sum of such functions in terms of their conjugates. Lemma 29 was established by Borwein in [5], Theorem 8.1, p. 421, and by Attouch-Brézis in [1], Theorem (1.1), Step 2, p. 129.

Lemma 29. Let $g_{1}, g_{2}: E \rightarrow \mathbb{R} \cup\{\infty\}$ be convex lower semicontinuous functions and $\operatorname{dom} g_{1}-\operatorname{dom} g_{2}$ be absorbing. Then

$$
\inf _{E}\left(g_{1}+g_{2}\right)+\min _{z_{1}^{*}+z_{2}^{*}=0} g_{1}^{*}\left(z_{1}^{*}\right)+g_{2}^{*}\left(z_{2}^{*}\right)=0 .
$$


Attouch-Brézis then deduce the following three results.

Theorem 30. (See [1], Theorem (1.1), Step 3, p. 129) Let $g_{1}, g_{2}: E \rightarrow \mathbb{R} \cup\{\infty\}$ be convex lower semicontinuous functions and

$$
\bigcup_{\lambda>0} \lambda\left[\operatorname{dom} g_{1}-\operatorname{dom} g_{2}\right]=\varlimsup
$$

Then (29.1) holds.

Corollary 31. (See [1], Corollary (2.1), p. 130) Let $f_{1}, f_{2}: E \rightarrow \mathbb{R} \cup\{\infty\}$ be convex lower semicontinuous functions and

$$
\bigcup_{\lambda>0} \lambda\left[\operatorname{dom} f_{1}-\operatorname{dom} f_{2}\right]=\overline{\operatorname{lin}\left(\operatorname{dom} f_{1}-\operatorname{dom} f_{2}\right)} .
$$

Then $\partial\left(f_{1}+f_{2}\right)=\partial f_{1}+\partial f_{2}$.

Corollary 32. (See [1], Corollary (2.2), p. 131) Let $g: F \rightarrow \mathbb{R} \cup\{\infty\}$ be a convex lower semicontinuous function, $Y$ be a closed subspace of $F, \inf _{Y} g \in \mathbb{R}$ and

$$
\bigcup_{\lambda>0} \lambda[\operatorname{dom} g+Y]=\overline{\operatorname{lin}(\operatorname{dom} g+Y)} \text {. }
$$

Then

$$
\inf _{Y} g+\min _{Y^{\perp}} g^{*}=0 .
$$

The passages from Lemma 29 to Theorem 30 and from Theorem 30 to Corollaries 31 and 32 are straightforward. However, the proof of Lemma 29 in [1] is not easy, and uses the Banach-Dieudonné-Krein-Smulian Theorem, and the proof of Lemma 29 in [5] depends on some technical results on convex processes. Here we shall show how to deduce a special case of Corollary 32 - Lemma 33 - directly from Theorem 3 using a simple geometric argument. We shall then show how to deduce Lemma 29 from Lemma 33. (Incidentally, one of the hypotheses originally given in [5], Theorem 8.1 was not sufficiently strong — see [22], p. 697 - but this problem does not affect the applications that we have in mind here.)

Lemma 33. Let $g: F \rightarrow \mathbb{R} \cup\{\infty\}$ be a convex lower semicontinuous function, $Y$ be a closed subspace of $F$, dom $g+Y$ be absorbing and $\inf _{Y} g=0$. Then

$$
\min _{Y^{\perp}} g^{*}=0 .
$$

Proof. We first note that if $x^{*} \in Y^{\perp}$, then

$$
g^{*}\left(x^{*}\right)=\sup _{F}\left(x^{*}-g\right) \geq \sup _{Y}\left(x^{*}-g\right)=\sup _{Y}(-g)=-\inf _{Y} g=0,
$$

so $\inf _{Y \perp} g^{*} \geq 0$. Thus it suffices for (33.1) to prove that

$$
\text { there exists } x^{*} \in Y^{\perp} \text { such that } g^{*}\left(x^{*}\right) \leq 0 .
$$

We write $A:=\operatorname{dom} g \times Y$ and define the function $f: A \times F^{*} \rightarrow \mathbb{R}$ by

$$
f\left((x, z), x^{*}\right):=g(x)+\left\langle z-x, x^{*}\right\rangle .
$$

From Theorem 3, there exist $n \geq 1$ and $\eta>0$ such that

$$
w \in F \text { and }\|w\| \leq \eta \quad \Longrightarrow \quad w \in F\{g \leq n\}+Y .
$$

We write $M:=n / \eta$, and we shall first prove that,

$$
\text { for all }(x, z) \in A, \quad g(x)+M\|z-x\| \geq 0 .
$$


So suppose that $(x, z) \in A$. If $\|z-x\|=0$ then $x \in Y$, and (33.4) is immediate. If, on the other hand, $\|z-x\|>0$, we write $\lambda:=\eta /\|z-x\|$. Then $\|\lambda z-\lambda x\|=\eta$, and hence, from (33.3), there exists $v \in F\{g \leq n\}$ such that $\lambda z-\lambda x \in v+Y$, from which

$$
\frac{\lambda x+v}{\lambda+1} \in Y
$$

Using the fact that $g(v) \leq n$, the convexity of $g$ and (33.5), we get

$$
\frac{\lambda g(x)+n}{\lambda+1} \geq \frac{\lambda g(x)+g(v)}{\lambda+1} \geq g\left(\frac{\lambda x+v}{\lambda+1}\right) \geq \inf _{Y} g=0,
$$

from which $g(x)+n / \lambda \geq 0$. (33.4) follows from this since

$$
\frac{n}{\lambda}=\frac{n}{\eta}\|z-x\|=M\|z-x\| \text {. }
$$

This completes the proof of (33.4). We now write

$$
B:=\left\{x^{*}:\left\|x^{*}\right\| \leq M\right\} \subset F^{*} .
$$

It now follows from (33.4) and the Hahn-Banach theorem that

$$
\inf _{A} \max _{B} f \geq 0 \text {. }
$$

$A$ and $B$ are convex. We now give $F^{*}$ the weak ${ }^{*}$ topology, and it then follows from the Banach-Alaoglu theorem that $B$ is compact. Further, $f$ is convex on $A$, and affine and continuous on $B$. Thus, from Theorem 14 ,

$$
\max _{B} \inf _{A} f \geq 0
$$

that is to say, there exists $x^{*} \in B$ such that

$$
\text { for all }(x, z) \in A, \quad g(x)+\left\langle z-x, x^{*}\right\rangle \geq 0 .
$$

This can be rewritten

$$
\text { for all }(x, z) \in A, \quad\left\langle x, x^{*}\right\rangle-g(x) \leq\left\langle z, x^{*}\right\rangle .
$$

Taking the supremum over $x$ and the infimum over $z$, we get

$$
g^{*}\left(x^{*}\right) \leq \inf _{Y} x^{*}
$$

Since the left hand side is $>-\infty$, we derive from this that $x^{*} \in Y^{\perp}$ and $g^{*}\left(x^{*}\right) \leq 0$. This completes the proof of (33.2).

New proof of Lemma 29. This follows from Lemma 33, using the substitutions $F:=$ $E^{2}, Y:=\{(z, z): z \in E\}$ and $g\left(x_{1}, x_{2}\right):=g_{1}\left(x_{1}\right)+g_{2}\left(x_{2}\right)$.

Corollary 34 is an easy consequence of Theorem 30:

Corollary 34. Let $m \geq 2, g_{1}, \ldots, g_{m}: E \rightarrow \mathbb{R} \cup\{\infty\}$ be convex lower semicontinuous functions and, for all $i=1, \ldots, m-1$,

$$
\bigcup_{\lambda>0} \lambda\left[\operatorname{dom} g_{i+1}-\bigcap_{j=1}^{i} \operatorname{dom} g_{j}\right]=\overline{\operatorname{lin}\left(\operatorname{dom} g_{i+1}-\cap_{j=1}^{i} \operatorname{dom} g_{j}\right)} .
$$

Then

$$
\inf _{E}\left(g_{1}+\cdots+g_{m}\right)+\min _{z_{1}^{*}+\cdots+z_{m}^{*}=0} g_{1}^{*}\left(z_{1}^{*}\right)+\cdots+g_{m}^{*}\left(z_{m}^{*}\right)=0
$$


We show finally how the results of this section lead to an improvement of a result that was shown to us by J. M. Borwein (personal communication) - Theorem 36 , but with the additional hypothesis that $E$ be reflexive. We start off with a consequence of Corollary 31.

Lemma 35. Let $E$ be a Banach space, $f_{1}, f_{2}: E \rightarrow \mathbb{R} \cup\{\infty\}$ be convex lower semicontinuous functions and

$$
\bigcup_{\lambda>0} \lambda\left[\operatorname{dom} f_{1}-\operatorname{dom} f_{2}\right]=\overline{\operatorname{lin}\left(\operatorname{dom} f_{1}-\operatorname{dom} f_{2}\right)} .
$$

Then $D\left(\partial f_{1}\right) \cap D\left(\partial f_{2}\right) \neq \emptyset$.

Proof. Clearly $D\left(\partial f_{1}\right) \cap D\left(\partial f_{2}\right)=D\left(\partial f_{1}+\partial f_{2}\right)$ and, from Corollary 31,

$$
D\left(\partial f_{1}+\partial f_{2}\right)=D\left(\partial\left(f_{1}+f_{2}\right)\right) .
$$

Since $\operatorname{dom} f_{1} \cap \operatorname{dom} f_{2} \neq \emptyset, f_{1}+f_{2}$ is proper, and, from the Brøndsted-Rockafellar theorem, $D\left(\partial\left(f_{1}+f_{2}\right)\right) \neq \emptyset$. This gives the desired result.

Theorem 36. Let $E$ be a Banach space and $g_{1}, g_{2}: E \rightarrow \mathbb{R} \cup\{\infty\}$ be convex lower semicontinuous functions such that $\operatorname{dom} g_{1}-\operatorname{dom} g_{2}$ is absorbing. Then

$$
D\left(\partial g_{1}\right)-D\left(\partial g_{2}\right) \text { is a neighborhood of } 0 .
$$

Proof. From Corollary 4, there exists $\eta>0$ such that

$$
w \in E \text { and }\|w\|<\eta \quad \Longrightarrow \quad w \in \operatorname{dom} g_{1}-\operatorname{dom} g_{2} .
$$

We shall show that

$$
v \in E \text { and }\|v\|<\eta \quad \Longrightarrow \quad v \in D\left(\partial g_{1}\right)-D\left(\partial g_{2}\right),
$$

which gives the desired result. So let $v \in E$ and $\|v\|<\eta$. Define $f_{1}, f_{2}: E \rightarrow$ $\mathbb{R} \cup\{\infty\}$ by $f_{1}(x):=g_{1}(x+v)$ and $f_{2}:=g_{2}$. If $u \in E$ and $\|u\|<\eta-\|v\|$, then $\|u+v\|<\eta$; hence, from (36.1), there exist $x_{i} \in \operatorname{dom} g_{i}$ such that $u+v=x_{1}-x_{2}$. Thus

$$
u=\left(x_{1}-x_{2}\right)-v=\left(x_{1}-v\right)-x_{2} \in \operatorname{dom} f_{1}-\operatorname{dom} f_{2} .
$$

So we have proved that $\operatorname{dom} f_{1}-\operatorname{dom} f_{2}$ is a neighborhood of 0 . From Lemma 35, there exists $x \in D\left(\partial f_{1}\right) \cap D\left(\partial f_{2}\right)$. But then $v=(x+v)-x \in D\left(\partial g_{1}\right)-D\left(\partial g_{2}\right)$. This completes the proof of (36.2).

Acknowledgement. The author would like to thank Professor Jonathan M. Borwein for reading earlier versions of this paper with enormous care, and making a number of extremely penetrating and useful comments and suggestions.

Remark added in press. Using Lemma 17, one can in fact prove that the conditions $(0.2),(0.3)$ are equivalent to the dom-dom constraint qualification (0.4), and that the conditions $(0.5),(0.6),(0.7)$ and $(23.1)$ are equivalent to the $\chi$ constraint qualification. Details of these results are in the addendum Pairs of monotone operators, which is the paper immediately following this one. 


\section{REFERENCES}

1. H. Attouch and H. Brézis, Duality for the sum of convex funtions in general Banach spaces, Aspects of Mathematics and its Applications, J. A. Barroso, ed., Elsevier Science Publishers, 1986, pp. 125-133. MR 87m:90095

2. H. Attouch, H. Riahi and M. Théra, Somme ponctuelle d'opérateurs maximaux monotones, Serdica 22 (1996), 165-190. CMP 97:14

3. J.-P. Aubin and I. Ekeland, Applied Nonlinear Analysis, Wiley, New York, 1984. MR 87a:58002

4. J. M. Borwein, A Lagrange multiplier theorem and a sandwich theorem for convex relations, Math. Scand. 48 (1981), 198-204. MR 83d:49027

5. J. M. Borwein, Adjoint process duality, Math. Oper. Res. 8 (1983), 403-434. MR 85h:90092

6. J. M. Borwein and S. Fitzpatrick, Local boundedness of monotone operators under minimal hypotheses, Bull. Australian Math. Soc. 39 (1988), 439-441. MR 90c:47093

7. H. Brézis, M. G. Crandall and A. Pazy, Perturbations of nonlinear maximal monotone sets in Banach spaces, Comm. Pure. Appl. Math. 23 (1970), 123-144. MR 41:2454

8. F. E. Browder, Nonlinear maximal monotone operators in Banach spaces, Math. Annalen 175 (1968), 89-113. MR 36:6989

9. M. Coodey and S. Simons, The convex function determined by a multifunction, Bull. Austral. Math. Soc. 54 (1996), 87-97. CMP 96:16

10. L.-J. Chu, On the sum of monotone operators, Michigan Math. J. 43 (1996), 273-289. CMP 96:15

11. K. Fan, Minimax theorems, Proc. Nat. Acad. Sci. U.S.A. 39 (1953), 42-47. MR 14:1109f

12. R. B. Holmes, Geometric functional analysis and its applications, Springer-Verlag, Graduate Texts in Mathematics, 24, New York-Heidelberg, 1975. MR 53:14085

13. H. König, Über das Von Neumannsche Minimax-Theorem, Arch. Math. 19 (1968), 482-487. MR 39:1947

14. R. R. Phelps, Convex Functions, Monotone Operators and Differentiability, Lecture Notes in Mathematics 1364 (Second Edition), Springer-Verlag, Berlin, 1993. MR 94f:46055

15. R. R. Phelps, Lectures on Maximal Monotone Operators, 2nd Summer School on Banach Spaces, Related Areas and Applications, Prague and Paseky, August 15-28, 1993. (Preprint, 30 pages.), TeX file: <math.okstate.edu/pub/banach/phelpsmaxmonop.tex > Banach space bulletin board archive, Posted Nov. 1993.

16. S. M. Robinson, Regularity and stability for convex multivalued functions, Math. Oper. Res. 1 (1976), 130-143; 2 (1977), 382. MR 55:3188; MR 57:12801

17. R. T. Rockafellar, Local boundedness of nonlinear, monotone operators, Michigan Math. J. 16 (1969), 397-407. MR 40:6229

18. R. T. Rockafellar, On the maximality of sums of nonlinear monotone operators, Trans. Amer. Math. Soc. 149 (1970), 75-88. MR 43:7984

19. S. Simons, Critères de faible compacité en termes du théorème de minimax, Seminaire Choquet, 1970/1971, Fasc. 2, Exposé 24, Secrétariat Math., Paris, 1971. MR 57:17218

20. S. Simons, The range of a monotone operator, J. Math. Anal. Appl. 199 (1996), 176-201. MR 97e: 47088

21. C. Ursescu, Multifunctions with convex closed graph, Czechoslovak Math. J. 25 (1975), 438441. MR 52:8869

22. C. Zălinescu, Letter to the editor: on J. M. Borwein's paper: "Adjoint process duality", Math. Oper. Res. 11 (1986), 692-698. MR 88h:90172

Department of Mathematics, University of California, Santa Barbara, California 93106-3080

E-mail address: simons@math.ucsb.edu 